\title{
Diagnostic precision of the Wisconsin Card Sorting Test in assessing cognitive deficits in substance use disorders
}

\section{B. Faustino, J. Oliveira \& P. Lopes}

To cite this article: B. Faustino, J. Oliveira \& P. Lopes (2021) Diagnostic precision of the Wisconsin Card Sorting Test in assessing cognitive deficits in substance use disorders, Applied Neuropsychology: Adult, 28:2, 165-172, DOI: 10.1080/23279095.2019.1607737

To link to this article: https://doi.org/10.1080/23279095.2019.1607737

\section{Published online: 06 May 2019.}

Submit your article to this journal

Џll Article views: 514

\section{Q View related articles ¿}

View Crossmark data $₫$

Citing articles: 3 View citing articles $\square$ 


\title{
Diagnostic precision of the Wisconsin Card Sorting Test in assessing cognitive deficits in substance use disorders
}

\author{
B. Faustino ${ }^{a, b}$ iD, J. Oliveira ${ }^{a}$ iD, and P. Lopes ${ }^{a}$

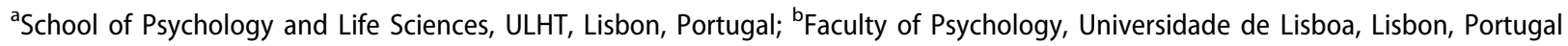

\begin{abstract}
The Wisconsin Card Sorting Test (WCST) is a widely used neuropsychological instrument to assess executive functions related to cognitive flexibility and abstract reasoning. However, there is a lack of studies investigating the diagnostic precision of this instrument in substance use disorders. In this study, we aimed at assessing the sensitivity and specificity of the WCST in discriminating the performance of participants with substance use disorder with cognitive deficits and participants from the general population without cognitive deficits. The sample comprised three groups of participants with substance use disorders (opioid use disorder in harm reduction with methadone maintenance; opioid use disorder in treatment in a therapeutic community; alcohol use disorder in a therapeutic community) and a normative group of healthy adults. The total sample consisted of 587 participants that were assessed with cognitive tests for executive functions, general cognitive functioning, and self-reported depression. The results showed differences between groups in most WCST variables, providing evidence of discriminant validity for this test. Convergent validity was also established by weak to moderate correlations with general cognitive functioning. Cutoff points based on receiver operating characteristic (ROC) curves were calculated for the WCST variables. Overall, the WCST was sensitive to changes in cognitive flexibility and abstract reasoning that are key features of substance use disorders.
\end{abstract}

\section{KEYWORDS}

Abstract reasoning; cognitive flexibility; executive functions; substance use disorders; Wisconsin Card Sorting Test

\section{Introduction}

The Wisconsin Card Sorting Test (WCST) is a neuropsychological assessment tool widely used in a variety of contexts, particularly in neurology, clinical neuropsychology, and psychiatry (Eling, Derckx, \& Maes 2008; Lezak, 2004). In the last four decades, the WCST was one of the most used neuropsychological instruments to assess frontal and prefrontal lobe dysfunction, which is shown by the growing number of publications on the use of the WCST (Eling, Derckx, \& Maes 2008; Nyhus \& Barceló, 2009).

WSCT is a test that allows assessing abstract reasoning and the ability to generate, modify, and apply a cognitive strategy in adaptation to changing environmental contingencies (Berg, 1948; Heaton, Chelune, Talley, Kay, \& Curtiss, 2005). According to Lezak (2004), the WSCT is used to evaluate the ability for deduction of concepts, to generate cognitive strategies, and to adapt behavior to changing conditions of the environment. Thus, this instrument is conceptualized as a measure of cognitive flexibility due to the mental operations underlying such cognitive processes, namely: (a) abstraction of an arbitrary rule according to a set of stimuli, (b) applying this rule according to environmental feedback, (c) generation of a new rule by modifying the previous rule, and (d) application of the new rule as a function of environmental feedback (Heaton et al., 2005).

The WCST has been also used to assess executive functioning (Zilmer, Spiers, \& Culbertson, 2008). Executive functions (EF) is an umbrella to refer to "higher-level" cognitive functions involved in the control and regulation of "lower-level" cognitive processes and goal-directed, future-oriented behavior (Alvarez \& Emory, 2006). According to Diamond (2013), the WCST is a measure of set-shifting, a compound of $\mathrm{EF}$, along with inhibition and working memory similar to the three-factor model of EF from Miyake et al. (2000).

In the field of clinical neuropsychology, there is agreement that individuals with epilepsy, autism spectrum disorder, and attention-deficit hyperactivity 
disorder, depressive disorder, and schizophrenia, show inferior performance in this test when compared to healthy individuals (Alvarez \& Emory, 2006; Diamond, 2013; Heaton et al., 2005).

There are also studies suggesting that individuals with substance use disorders (SUD) show greater deficits in executive functions when compared with healthy individuals (Bruijnen et al., 2019). For instance, it was found that cocaine users have higher perseverance rates in decision tests, lower working memory capacity and poor general cognitive flexibility in comparison to healthy controls (Rosselli, Ardila, Lubomski, Murray, \& King, 2001; Rosselli \& Ardila, 1996; Vonmoos et al., 2014; Woicik et al., 2009). According to other authors, heroin users seem to have an impairment in impulse control, but not in the attentional processes and cognitive flexibility (Pau, Lee, \& Chan, 2002).

Alcohol abuse disorder has been also associated with deficits at the level of executive functions, particularly in working memory, set shifting, and inhibition abilities (Dry, Burns, Nettelbeck, Farquharson, \& White, 2012; Park et al., 2011; Spinola, Maisto, White, \& Huddleson, 2017). Moreover, Inozemtseva, PerezSolis, Matute, and Juarez (2016) and Hagen et al. (2017) showed that patients with SUD improve their level of cognitive and executive functioning measured with the WCST, following one-year of abstinence. Other authors suggest that overall cognition of individuals in methadone maintenance treatment is largely intact, but cognitive deficits in SUD may arise from other psychiatric comorbidities, duration of substance abuse and the type of substance (Elkana, Adelsom, Doniger, Sason, \& Peles, 2017).

According to Fernández-Serrano, Pérez-García, and Verdejo-García (2011), drug abuse is commonly associated with changes in neuropsychological domains of episodic memory, emotional processing, as in executive components of updating and decision-making. Also, specific neuropsychological impairments due to psychostimulants and alcohol abuse have been found on impulsive action and cognitive flexibility, whereas alcohol and 3,4-Methyl enedioxy methamphetamine (MDMA) abuse may impact on spatial processing, perceptual speed and selective attention, cannabis and methamphetamine abuse on prospective memory deficits, and cannabis and MDMA abuse on processing speed and complex planning (Fernández-Serrano et al., 2011). Overall effects may be attenuated by achieving long-term abstinence, but opioid-related effects on updating and decision-making may endure beyond long-term periods. In this sense, more studies comparing different SUD groups at distinct stages of treatment may shed light on the neuropsychological effects of substance abuse. Thus, it is also not clear whether the WCST is sensitive to the effects that may be specific of the type of substance or that may be more pronounced at short-term abstinence.

A meta-analysis on sensitive neuropsychological measures for alcohol effects concluded that the Hayling Test, WCST, and the Iowa Gambling Task were the most sensitive tests to detect changes in planning, problem-solving, and inhibitory abilities resulting from alcohol abuse (Stephan et al., 2017). These researchers suggest that alcohol abuse is associated with executive dysfunction, but a recent study of Martins, Bartholow, Cooper, Von Gunten, and Wood (2018) with individuals from the general population, failed to provide consistent evidence for the role of executive function abilities on alcohol use, heavy drinking, and alcohol-related behaviors.

Given these findings, this study was developed to explore whether the WCST may be sensitive to the cognitive changes resulting from substance abuse. It is known that the prefrontal cortex is particularly vulnerable to the effects of substance use (such as alcohol, or heroin), which may compromise decisionmaking processes, as well as the underlying cognitive abilities as working memory. This study aimed thus to provide evidence for discriminant and convergent validity, along with the cutoffs according to sensitivity and specificity in detecting executive dysfunction, as well as the normative scores for the WCST in these populations. Specificity and sensitivity were used according to the Signal Detection Theory (SDT), which are based on statistical procedures to study the criterion of the observer (decision-making) in detecting the "signal" under conditions of uncertainty (Van der Kellen, Nunes, \& Garcia-Marques, 2008). Considering that the tasks implemented in WCST are classification tasks that require decision-making abilities subjected to error/noise, in our view the SDT is appropriate to assess discrimination performance of observers/participants the tasks. The main objectives of this study are as follows: (a) test whether there are differences in the WSCT scores for nonclinical versus clinical groups to provide evidence of discriminant validity in SUD; (b) identify the relationships between the WCST with global cognitive functioning, executive functions, and working memory abilities to provide evidence of convergent validity in SUD; and (c) identify the best cutoffs to distinguish between clinical vs. nonclinical scores in the WCST according to sensitivity and specificity ratings in discriminating SUD. 
Table 1. Sociodemographic characterization of the total sample $(n=587)$.

\begin{tabular}{lccr}
\hline Variables & Categories & $n$ & $\%$ \\
\hline Age $(M=42.1 ; S D=10.4)$ & $15-19$ & 5 & 0.9 \\
& $20-29$ & 60 & 10.3 \\
& $30-39$ & 185 & 32.7 \\
& $40-49$ & 197 & 33.8 \\
& $50-59$ & 106 & 18.2 \\
& $60-69$ & 28 & 4.8 \\
Gender & $70-79$ & 2 & 0.3 \\
& Male & 422 & 71.9 \\
Years of Study & Female & 165 & 28.1 \\
& O years & 19 & 3.2 \\
& $1-4$ years & 56 & 9.5 \\
& $5-9$ years & 172 & 29.3 \\
& $10-12$ years & 172 & 29.3 \\
& $13-15$ years & 96 & 16.4 \\
Group & $16-18$ years & 44 & 7.5 \\
& More de 18 years & 28 & 4.8 \\
& HA & 182 & 31.0 \\
& OUDMM & 111 & 18.9 \\
& OUD & 118 & 20.1 \\
& AUD & 176 & 30.0 \\
\hline
\end{tabular}

Note. $\mathrm{HA}=$ healthy adults; OUDMM $=$ opioid use disorder in methadone maintenance; OUD = opioid use disorder; AUD = alcohol use disorder.

\section{Method}

\section{Participants}

The sample was based on archival data from 587 individuals (422 men, 71.9\%) with age ranged between 19 to 73 years $(M=42.1 ; S D=10.4)$. This sample comprised four different groups: three groups of patients with SUD: one group of patients undergoing community treatment for alcohol use disorder in abstinence-AUD ( $n=176, M=46.9 ; S D=7.8)$; one group of patients undergoing community treatment for opioid use disorder in abstinence OUD $(n=118$; $M=40.5 ; S D=8.8$; ; and one group of individuals with OUD in harm reduction with methadone maintenance OUDMM $(n=111 ; M=40.5 ; S D=8.1)$. Another group of healthy adults: HA $(n=182 ; M=$ $40.5 ; S D=12.5$ ) matched for gender and education was also included (Table 1).

The groups undergoing therapeutic treatment for SUD in a therapeutic community were in abstinence. These subjects are mainly polysubstance users in which the substance of preference is alcohol in AUD patients and heroin in OUD patients. Both were required to maintain in abstinence during treatment. Abstinence was ensured by urinary analysis for each of the metabolites from these substances. This was an inclusion criterion for this study. Most of these patients were medicated with stable medication regimens that consisted of benzodiazepines and antidepressives to reduce deprivation. As regards the OUDMM, these patients were integrated into a low threshold methadone maintenance program in which abstinence was not required. Only patients that they had a stable methadone dosage in the last 30 days before the evaluation were considered for this study. This information was available from the archival data at the time of the assessment that was conducted to these patients.

The general inclusion criteria for this study were: (a) being able to read and write in Portuguese irrespective of schooling years; (b) Portuguese nationality; (c) aged over 15 years, (d) clinical diagnosis of SUD (alcohol or opioids), and (e) being enrolled in a treatment facility (patients in abstinence assessed through urinary analysis) or harm reduction program (patients with stable methadone dosage in the last 30 days before assessment). The exclusion criterion was having any psychiatric or mental disorder other than those specified for each clinical group.

\section{Measures}

\section{Wisconsin Card Sorting Test}

The WCST (Heaton et al., 2005) is a widely used neuropsychological test to assess executive functions, cognitive flexibility, and abstract reasoning. The WCST is scored on eleven performance parameters, namely: NTA $=$ No. of trials administered; NTE $=$ No. of total errors; NP $=$ No. of perseverations; NPE $=$ No. of perseverative errors; NNPE $=$ No. of nonperseverative errors; CLR $=$ Conceptual level responses; NCC $=$ No. of categories completed; NTCF $=$ No. of trials to complete first category; FTMS = Failure to maintain set; $\mathrm{OR}=$ Other response. These indexes were computed in percentages.

\section{Frontal Assessment Battery (FAB)}

The Frontal Assessment Battery (FAB; Dubois, Slachevsky, Litvan, \& Pillon, 2000; Portuguese version of Lima et al., 2008) is a screening instrument for the assessment of executive dysfunction. This battery comprises six subtests that evaluate brain processes typically associated with frontal lobe functioning: Similarities (conceptualization), Lexical fluency (mental flexibility), Motor series "Luria" test (programming), conflicting instructions (sensitivity to interference), Go-No-Go (inhibitory control), and (environmental autonomy). Each subtest is scored between $0-3$, with total scores ranging $0-18$.

\section{Mini-Mental State Examination (MMSE)}

The Mini-Mental State Examination (MMSE, Folstein, Folstein, \& McHugh, 1975; Portuguese version of Guerreiro, Silva, \& Botelho, 1994) is a widely used cognitive screening test. The MMSE consists of 11 
items, divided into two sections. The first requires verbal responses to questions of orientation, memory, and attention. The second section requires reading and writing skills, following verbal and written commands, as writing a sentence and copying drawing abilities (polygons). The questions are scored by adding an immediate score to each task that is completed successfully, which ranges between $0-30$ for the total score.

\section{Digit Memory Task of the Wechsler Memory Scale (WMS)}

The Reverse Digit Span is a subtest of the Wechsler Memory Scale - 3rd Edition (WMS - III; Wechsler, 2008). This task consists in repeating in direct and reverse order the series of digits, of increasing size, and distributed by items. Each item corresponds to a different sequence length. The task ends when the subject fails two trials on the same sequence. The total score consists of the maximum number of correctly repeated sequences. The score ranges from 0 to 14 , in which higher scores are associated with better the working memory ability (Wechsler, 2008).

\section{Beck Depression Inventory (BDI)}

The Beck Depression Inventory (BDI; Beck, Ward, Mendelson, Mock, \& Erbaugh, 1961; Portuguese version of Vaz-Serra \& Abreu, 1973) is a 21-item selfreport inventory that measures the severity of symptoms associated with depression. For each item, participants select between four scores, ranging from 0 (the symptom is not present) to 3. The overall BDI score is given by the sum of all items, which ranges from 0 to 63 .

\section{Procedure}

The data was collected from the clinical files from specialized institutions for treatment of SUD. The sample of patients with AUD was collected at Casa de Saúde do Telhal, which is a rehabilitation institution for alcohol dependence treatment; whereas, the sample of patients with OUD was collected at Ares do Pinhal, which is an NGO rehabilitation institution for treatment of drug addiction, both institutions in Lisbon region of Portugal. The normative group data was collected from a prior research project from Faculty of Psychology and Life Sciences of University Lusofona in Lisbon. The data were standardized by calculating percentages to WCST indexes, and mean scores for other neuropsychological variables. Test administration order was not controlled, due to the fact that the data was collected at different assessment moments and/or research projects.

\section{Statistical analysis}

The statistical analysis was performed with the Statistical Package for the Social Sciences (IBM SPSS Statistics, version 24.0 for Windows). Descriptive statistics was used for sample characterization. Firstly, the analysis of covariance (ANCOVA) was conducted for each of the WCST parameters to provide evidence of discriminant validity. This analysis was conducted using group and education as factors in the analysis, while age was controlled as a covariate in this analysis. The significant tests in the ANCOVA were explored further with post-hoc analysis with Bonferroni comparisons. The convergent validity was tested with Pearson product-moment correlations to explore the relationships between WCST scores with global cognitive functioning (MMSE), executive functions (FAB) and working memory (Digit memory task - WMS III). Finally, the diagnostic ability of the WCTS in detecting the differences between groups was tested with the receiver operating characteristic (ROC) curves. The best cutoff values were calculated for maximum sensitivity and specificity ratings.

\section{Results}

\section{Discriminant validity}

This analysis was conducted through the ANCOVA to test whether there were differences between groups for each index of the WCST, while controlling for education and age effects. Education was included in the analysis as a factor while age was controlled as a covariate.

Table 2 describes the ANCOVA for the 10 WCST indexes. The ANCOVA showed that most of the indexes of the WCTS distinguished the study groups, except for NNPE, NTFC, FTMS and OR that were not statistically significant $(p>.05)$. No significant interaction effects were found with education ( $p$ $>.05)$.

The post hoc comparisons showed differences between HA versus OUD only in NTA $(p<.05)$, while between HA versus AUD, differences were found in NTA, NTE, CLR, and NCC $(p<.05)$. AUD had higher scores that HA in NTA and NTE and lower scores in NCC and CLR. Also, differences between AUD versus OUD and AUD versus OUDMM were found in NTA, NTE, NP, CLR, and NCC $(p<.05)$, while between AUD versus OUDMM 
Table 2. ANCOVA for the 10 WCST indexes.

\begin{tabular}{|c|c|c|c|c|c|c|c|c|c|c|}
\hline & NTA & NTE & $\mathrm{NP}$ & NPE & NNPE & CLR & NCC & NTCF & FTMS & $\mathrm{OR}$ \\
\hline$F$ & $9.700^{* * * *}$ & $6.761^{* * *}$ & $3.734 * * *$ & $3.902 * * *$ & 2.065 & $4.885^{* * *}$ & $6.135^{* * *}$ & 0.672 & 0.603 & 1.023 \\
\hline Fgroup $\times$ education & 0.119 & 0.387 & 0.740 & 0.726 & 0.759 & 0.089 & 0.291 & 0.438 & 0.351 & 0.964 \\
\hline
\end{tabular}

Note. ANCOVA $=$ Analysis of covariance; WCST $=$ Wisconsin Card Sorting Test; NTA $=$ No. of trials administered; NTE $=$ No. of total errors; NP $=$

No. of perseverations; NPE $=$ No. of perseverative errors; NNPE $=$ No. of non-perseverative errors; $C L R=$ Conceptual level responses; NCC $=$

No. of categories completed; NTCF $=$ No. of trials to complete first category; FTMS = Failure to maintain set; OR $=$ Other response.

$* * * p<.001$.

Table 3. Person correlations between 10 WSCT indexes with MMSE, FAB, BDI, and WMS.

\begin{tabular}{lcccc}
\hline & MMSE & FAB & BDI & WMS - Reversed digits \\
\hline NTA & $-0.302^{* *}$ & $-0.327^{* *}$ & $0.202^{* *}$ & $-0.238^{* *}$ \\
NTE & $-0.464^{* *}$ & $-0.500^{* *}$ & $0.232^{* *}$ & $-0.215^{* *}$ \\
NP & $-0.376^{* *}$ & $-0.451^{* *}$ & $0.178^{* *}$ & $-0.173^{* *}$ \\
NPE & $-0.398^{* *}$ & $-0.435^{* *}$ & $0.200^{* *}$ & $-0.120^{*}$ \\
NNPE & $-0.290^{* *}$ & $-0.299^{* *}$ & $0.181^{* *}$ & $-0.107^{*}$ \\
CLR & $0.377^{* *}$ & $0.340^{* *}$ & $-0.201^{* *}$ & $0.205^{* *}$ \\
NCC & $0.322^{* *}$ & $0.308^{* *}$ & -0.004 & $0.238^{* *}$ \\
NTCF & $-0.273^{* *}$ & $-0.260^{* *}$ & 0.097 & -0.093 \\
FTMS & -0.045 & -0.031 & 0.041 & -0.061 \\
OR & $-0.229^{* *}$ & $-0.267^{* *}$ & 0.035 & $-0.104^{*}$ \\
\hline WCST & & &
\end{tabular}

WCST $=$ Wisconsin Card Sorting Test; MMSE $=$ Mini-Mental State Examination; $\mathrm{FAB}=$ Frontal Assessment Battery; $\mathrm{BDI}=$ Beck Depression Inventory; WMS $=$ Wechsler Memory Scale; NTA $=$ No. of trials administered; NTE $=$ No. of total errors; NP $=$ No. of perseverations; NPE $=$ No. of perseverative errors; NNPE $=$ No. of non-perseverative errors; $\mathrm{CLR}=$ Conceptual level responses; NCC $=$ No. of categories completed; NTCF $=$ No. of trials to complete first category; FTMS $=$ Failure to maintain set; $\mathrm{OR}=$ Other response.

$*^{*} p<.01 .{ }^{*} p<.05$.

were found only in NPE $(p<.05)$. AUD had higher scores in all indexes, except for NNC.

\section{Convergent validity}

Pearson product-moment correlations between the 10 WCST indexes and the total values of the FAB, MMSE, BDI, and the WMS reversed digits were calculated to understand whether performance in the WCST correlates with measures of global cognitive functioning, executive functions, working memory, and self-reported depression. This analysis showed that most indexes of the WCST are related to global cognitive functioning, executive functions, working memory, but also to self-reported depression. Table 3 describes the coefficients and the statistical significance for each correlation.

\section{ROC analysis}

The ROC curves were used to identify the best cutoff scores for the eight WCST indexes in which significant differences were found between groups. Each clinical group was compared with the normative group. Thus, three ROC curves were computed, one for the comparison between OUDMM versus normative group, another for OUD in therapeutic
Table 4. ROC scores and cutoffs for the best WCST indexes in discriminating AUD group vs. healthy adults.

\begin{tabular}{lcccccccc}
\hline & AUC & SE & $p$-value & Max & Min & Cutoffs & Sensitivity & Specificity \\
\hline NTA & 0.719 & 0.028 & $<.001$ & 0.665 & 0.774 & 95.0 & 0.719 & 0.681 \\
NTE & 0.782 & 0.025 & $<.001$ & 0.773 & 0.831 & 27.0 & 0.766 & 0.709 \\
NP & 0.737 & 0.027 & $<.001$ & 0.684 & 0.791 & 14.0 & 0.725 & 0.654 \\
NPE & 0.753 & 0.026 & $<.000$ & 0.701 & 0.805 & 14.0 & 0.708 & 0.703 \\
NNPE & 0.708 & 0.028 & $<.000$ & 0.654 & 0.763 & 14.0 & 0.653 & 0.676 \\
CLR & 0.719 & 0.028 & $<.000$ & 0.664 & 0.774 & 60.0 & 0.719 & 0.670 \\
NCC & 0.767 & 0.026 & $<.000$ & 0.717 & 0.818 & 50.5 & 0.731 & 0.714 \\
NTCF & 0.749 & 0.027 & $<.000$ & 0.696 & 0.801 & 10.0 & 0.754 & 0.692 \\
\hline Note. ROC = receiver operating characteristic; WCST = Wisconsin Card \\
Sorting Test; AUD = alcohol use disorder; AUC = Area under the curve; \\
NTA = No. of trials administered; NTE = No. of total errors; NP = No. \\
of perseverations; NPE = No. of perseverative errors; NNPE = No. of \\
non-perseverative errors; CLR = Conceptual level responses; NCC = No. \\
of categories completed; NTCF = No. of trials to complete \\
first category.
\end{tabular}

community versus healthy adults, and finally for the comparison between AUD versus the normative group. Table 4 depicts the area under the curve (AUC), as well as the cutoffs identified for maximum sensitivity and specificity in the eight WCST indexes. The AUC values are satisfactory in all these indexes (Table 4).

\section{Discussion}

Despite the popularity of WCST as an instrument for assessing cognitive flexibility, abstract reasoning, and executive functions (Heaton et al., 2005; Lezak, 2004), to our knowledge the diagnostic ability in discriminating executive dysfunction in substance use disorder has not yet been tested prior to this study. Thus, the current study aimed to contribute to this topic by providing the data regarding the discriminant and convergent validity as well as the diagnostic ability of executive dysfunction according to the WCST in substance use disorders.

The results suggested differences in most of the WCTS indexes, with exception for the Failure to maintain a set (FTMS), Other response (OR), and No. of categories completed (NCC), which were excluded from further analysis. It is possible that these indexes do not directly depend on the neurocognitive characteristics of such individuals, not being sensitive to the cognitive changes associated with substance use 
disorders. However, the results showed that in the significant indexes, the group of patients with AUD showed higher scores in NTA and NTE and lower scores in NCC and CLR, than the group of healthy adults. These results suggest that frontal lobe functioning and cognitive flexibility may be more compromised due to alcohol effects than due to other substances such as heroin (Pau et al., 2002). This could explain the differences in the AUD and OUDMM groups in the WCST indexes, even considering that OUDMM patients were in a methadone substitution program receiving methadone during the time of assessment, although only patients receiving stable methadone dosage for more than 30 days were included in the study. These results concur with Davis, Liddiard, and McMillan (2002) suggesting that recovery of neuropsychological performance may be observed once a patient is stabilized in methadone, or Mintzer and Stitzer's (2002) results that found similar performance in patients using methadone compared to drug-free controls on executive functions.

Hagen et al. (2017) emphasize also that abstinence could reverse the neurocognitive deficits caused by substance abuse, while increasing functional abilities of these patients. One could argue that the differences between groups in the WCST can be also explained by the duration of abstinence. Elkana et al. (2017), states also that the duration of abstinence may have a positive effect in neurocognitive recovering, which is explained by the absence of nocive substances in the brain. However, the duration of abstinence was not controlled in this study, which is a limitation to these findings.

The ROC curves were used to identify the cutoff points in the eight significant indexes. Differences in these WCST indexes were also found in substance abuse disorders (Bruijnen et al., 2019), cocainedependent individuals (Vonmoos et al., 2014), and heavy drinking behaviors (Martins et al., 2018). In this sense, our data supports the notion of cognitive flexibility deficits in individuals with SUD.

The correlations with the MMSE, FAB, BDI, and the WMS (inverse digits) are also of interest. The negative correlations between the indexes related to poor cognitive flexibility (e.g., NTE; NP) were associated with general measures of cognition and executive functions, respectively, by the MMSE and the FAB. Conversely, the CLR index was positively associated with MMSE and FAB, which supports the notion that individuals with higher mental conceptualization skills show better cognitive abilities (Elkana et al., 2017).
Concerning working memory (WMS inverse digits), the associations were weak to moderate. These results suggest that the executive process of updating working memory is different from the process of cognitive flexibility, even though they are associated. This finding is in line with previous studies (Diamond, 2013, Miyake et al., 2000; Stuss \& Knight, 2013). Some authors suggest a conceptualization of EF, as a set of different but associated processes, such as cognitive flexibility, updating, and inhibition (Miyake \& Friedman, 2012).

Associations between depressive symptomatology and the WCST indexes were also found. The total score of the BDI was positively correlated with the NTA, NTE, NP, NPE, NNPE, and negatively associated with CLR, showing that depressive symptoms are associated with the number of errors, perseverations and the low-level of conceptual responses, suggesting that depressive symptomatology is related to poorer performance in the WCST. This is consistent with previous findings where depressive comorbidity to SUD was associated with poorer shifting abilities and perseverative errors (Heaton et al., 2005). Depression is associated with frontal lobe dysfunction and may contribute to deficits in cognitive flexibility and abstract reasoning as suggested by Merriam, Thase, Haas, Keshavan, and Sweeney (1999).

However, there are also some limitations to these results. The data were collected from the clinical files in each of these institutions and due to the different methodologies of data collection no uniform standard for the records and for test administration order. Thus, an extensive work was required to standardize the data in the different databases. This limitation was also related to the absence of data in some variables of the WCST. Another limitation was the discrepancy between the study's groups. In addition to the differences in sample size, there were statistically significant differences in age that was not possible to match between the samples. Although the potential effects of age and education on the WCST were accounted in the statistical analyses by including these variables as factors and covariates in the analyses. Furthermore, the temporal stability of the WCST would have been also important to understand how these scores change in a given time period.

In sum, the WCST may be sensitive to cognitive flexibility, abstract reasoning, and executive functions in populations with substance use disorders of alcohol or opioids. However, further research is needed with the WCST on other samples with substance use disorder to better understand the usefulness of the 
WCST in discriminating executive dysfunction due to substance abuse as to determine the specific effects of the substance on executive functioning.

\section{ORCID}

B. Faustino (D) http://orcid.org/0000-0002-7948-1622

J. Oliveira (D) http://orcid.org/0000-0002-3467-4981

P. Lopes (iD) http://orcid.org/0000-0002-3128-1365

\section{References}

Alvarez, J. A., \& Emory, E. (2006). Executive function and the frontal lobe: A meta-analytic review. Neuropsychology Review, 16(1), 17-42. doi:10.1007/s11065-006-9002-x

Ashendorf, L., \& McCaffrey, R. J. (2008). Exploring agerelated decline on the Wisconsin Card Sorting Test. The Clinical Neuropsychologist, 22(2), 262-272. doi:10.1080/ 13854040701218436

Berg, E. A. (1948). A simple objective technique for measuring flexibility in thinking. Journal of General Psychology, 39(1), 15-12. doi:10.1080/00221309.1948.9918159

Beck, A. T., Ward, C. H., Mendelson, M., Mock, J., \& Erbaugh, G. (1961). An inventory for measuring depression. Archives of General Psychiatry, 4, 53-63.

Bruijnen, C. J., Dijkstra, B. A., Walvoort, S. J., Markus, W., VanDerNagel, J. E., Kessels, R. P., \& De Jong, C. A. (2019). Prevalence of cognitive impairment in patients with substance use disorder. Drug Alcohol Review.

Darke, S., Sims, J., McDonald, S., \& Wickes, W. (2000). Cognitive impairment among methadone maintenance patients. Addiction, 95(5), 687-695. doi:10.1046/j.13600443.2000.9556874.x

Davis, P., Liddiard, H., \& McMillan, T. (2002). Neuropsychological deficits and opiate abuse. Drug and Alcohol Dependence, 67(1), 105-108. doi:10.1016/S03768716(02)00012-1

Diamond, A. (2013). Executive functions. Annual Review of Psychology, 64(1), 135-136. doi:10.1146/annurev-psych113011-143750

Dubois, B., Slachevsky, A., Litvan, I., \& Pillon, B. (2000). The FAB: A frontal assessment battery at bedside. Neurology, 55(11), 1621-1626.

Dry, M. J., Burns, N. R., Nettelbeck, T., Farquharson, A. L., \& White, J. M. (2012). Dose-related effects of alcohol on cognitive functioning. PLoS One, 7(11), e50977. doi: 10.1371/journal.pone.0050977

Eling, P., Derckx, K., \& Maes, R. (2008). On the historical and conceptual background of the Wisconsin Card Sorting Test. Brain and Cognition, 67(3), 247-253. doi: 10.1016/j.bandc.2008.01.00

Elkana, O., Adelsom, M., Doniger, G. M., Sason, A., \& Peles, E. (2017). Cognitive function is largely intact in methadone maintenance treatment patients. The World of Biological Psychiatry, 5, 1-11. doi:10.1080/15622975. 2017.1342047

Fernández-Serrano, M. J., Pérez-García, M., \& VerdejoGarcía, A. (2011). What are the specific vs. generalized effects of drugs of abuse on neuropsychological performance? Neuroscience Biobehavioral Review, 35(3), 377-406. doi:10.1016/j.neubiorev.2010.04.008

Folstein, M. F., Folstein, S. E., \& McHugh, P. R. (1975). Mini-mental state. Journal of Psychiatry Resources, 12(3), 189-198. doi:10.1016/0022-3956(75)90026-6

Guerreiro, M., Silva, A. P., \& Botelho, M. A. (1994). Adaptação à população Portuguesa na tradução do "Mini Mental State Examination" (MMSE) [Adaptation to the Portuguese population in the translation of the "Mini Mental State Examination" (MMSE)]. Revista Portuguesa de Neurologia, 1, 9-10.

Hagen, E., Erga, A. H., Hagen, H. P., Nesvag, S. M., Mckay, J. K., Lundervold, A. J., \& Walderhaug, E. (2017). Oneyear sobriety improves satisfaction with life, executive functions and psychological distress among patients with polysubstance use disorder. Journal of Substance Abuse Treatment, 76, 81-87. doi:10.1016/j.jsat.2017.01.016

Heaton, R. K., Chelune, G. J., Talley, J. L., Kay, G. G., \& Curtiss, G. (2005). Teste Wisconsin de Classificação de Cartas: manual revisado e ampliado. Adaptação e padronização brasileira Jurema Alcides Cunha et al. (2005). [Wisconsin Card Sorting Test: Revised and expanded manual. Adaptation and Brazilian Standardization] São Paulo: Casa do Psicólogo.

Inozemtseva, O., Perez-Solis, L., Matute, E., \& Juarez, J. (2016). Differential improvement of executive functions during abstinence in cocaine-dependent patients: A longitudinal study. Substance Use Misuse, 5(1), 1428-1440. doi:10.1080/10826084.2016.1178293

Lezak, M. D. (2004). Neuropsychological Assessment (4th ed.). New York, USA: Oxford University Press.

Lima, C. F. Meireles, L. P. Fonseca, R. Castro, S. L. \& Garrett, C. (2008). The Frontal Assessment Battery (FAB) in Parkinson's disease and correlations with formal measures of executive functioning. Journal of Neurology, 255(11), 1756-1761.

Martins, J. S., Bartholow, B. D., Cooper, M. L., Von Gunten, C. D., \& Wood, P. K. (2018). Associations between executive functioning, affect-regulation drinking motives, and alcohol use and problems. Psychology of Addictive Behaviors, 32(1), 16-28. doi:10.1037/ adb0000324

Merriam, E. P., Thase, M. E., Haas, G., Keshavan, M. S., \& Sweeney, J. A. (1999). Prefrontal cortical dysfunction in depression determined by Wisconsin Card Sorting Test performance. American Journal of Psychiatry, 156, 780-782.

Mintzer, M., \& Stitzer, M. (2002). Cognitive impairment in methadone maintenance patients. Drug and Alcohol Dependence, 67(1), 41-51. doi:10.1016/S03768716(02)00013-3

Miyake, A., Friedman, N. P., Emerson, M. J., Witzki, A. H., Howerter, A., \& Wager, T. D. (2000). The unity and diversity of executive functions and their contributions to complex "Frontal Lobe" tasks: A latent variable analysis. Cognitive Psychology, 41(1), 49-100. doi:10.1006/ cogp.1999.0734

Miyake, A., \& Friedman, N. P. (2012). The nature and organization of individual differences in executive functions: Four general conclusions. Current Directions in Psychological Science, 21(1), 8-14. doi:10.1177/ 0963721411429458 
Nyhus, E., \& Barceló, F. (2009). The Wisconsin Card Sorting Test and the Cognitive Assessment of Prefrontal Executive Functions: A critical update. Brain and Cognition, 71(3), 437-451. doi:10.1016/j.bandc.2009. 03.005

Park, M. S., Sohn, S., Park, J. E., Kim, S. H., Yu, I. K., \& Sohn, J. H. (2011). Brain functions associated with verbal working memory tasks among young males with alcohol use disorders. Scandinavian Journal of Psychology, 52(1), 1-7. doi:10.1111/j.1467-9450.2010.00848.x

Pau, C. W., Lee, T. M., \& Chan, S. F. (2002). The impact of heroin on frontal executive functions. Archives of Clinical Neuropsychology, 17(7), 663-670. doi:10.1093/ arclin/17.7.663

Rosselli, M., \& Ardila, A. (1996). Cognitive effects of cocaine and polydrug abuse. Journal of Clinical and Experimental Neuropsychology, 18(1), 122-135. doi: $10.1080 / 01688639608408268$

Rosselli, M., Ardila, A., Lubomski, M., Murray, S., \& King, K. (2001). Personality profile and neuropsychological test performance in chronic cocaine-abusers. The International Journal of Neuroscience, 110, 55-72. doi: 10.3109/00207450108994221

Stephan, R. A., Alhassoon, O. M., Allen, K. E., Wollman, S. C., Hall, M., Thomas, W. J., ... Grant, I. (2017). Meta-analyses of clinical neuropsychological tests of executive dysfunction and impulsivity in alcohol use disorder. The American Journal of Drug and Alcohol Abuse, 43(1), 24-43. doi:10.1080/00952990.2016.1206113

Spinola, S., Maisto, S. A., White, C. N., \& Huddleson, T. (2017). Effects of acute alcohol intoxication on executive functions controlling self-regulated behavior. Alcohol, 61, 1-8. doi:10.1016/j.alcohol.2017.02.177
Stuss, D., \& Knight, R. T. (2013). Principles of frontal lobe function (2nd ed.). New York, USA: Oxford University Press.

Van der Kellen, D., Nunes, L. D., \& Garcia-Marques, L. (2008). Sensibilidade e bom senso: Princípios fundamentais de deteção de sinal na investigação em Psicologia [Sensitivity and common sense: Fundamental principles of signal detection in research in psychology]. Laboratório de Psicologia, 6(1), 75-91.

Vaz-Serra, A., \& Abreu, J. (1973). Aferição dos quadros clínicos depressivos I: Ensaio de aplicação do "Inventário Depressivo de Beck" a uma amostra portuguesa de doentes deprimidos [Assessment of depressive clinical conditions I. "Beck Depressive Inventory." application test for a Portuguese sample of depressed patients]. Coimbra Médica, 20, 623-644.

Vonmoos, M., Hulka, L. M., Preller, K. H., Minder, F., Baumgartner, M. R., \& Quednow, B. B. (2014). Cognitive impairment in cocaine users is drug-induced but partially reversible: Evidence from a longitudinal study. Neuropsychopharmacology, 39(9), 2200-2210. doi:10.1038/ npp. 2014.71

Wechsler, D. (2008). WMS - Escala Memória de Wechsler Terceira Edição (3a Edição). Lisboa: CEGOC - TEA. [Wechsler Memory Scale].

Woicik, P. A., Moeller, S. J., Alia-Klein, N., Maloney, T., Lukasik, T. M., Yeliosof, O., ... Goldstein, R. Z. (2009). The neuropsychology of cocaine addiction: Recent cocaine use masks impairment. Neuropsychopharmacology, 34(5), 1112-1122. doi:10.1038/ npp. 2008.60

Zilmer, E. A., Spiers, M. V., \& Culbertson, W. C. (2008). Principles of neuropsychology (2nd ed.). Belmont, CA: Thomson Wadsworth. 